\title{
Targeting Obesity in Rural and Appalachian Children and Families: A Systematic Review of Prevention and Treatment Interventions
}

\author{
Melinda J. Ickes", Kira M. Slagle \\ Department of Kinesiology and Health, Promotion, University of Kentucky, Lexington, 40506, Kentucky, United States \\ *Corresponding author: melinda.ickes@uky.edu
}

Copyright (C) 2013 Horizon Research Publishing All rights reserved.

\begin{abstract}
Children living in rural and Appalachian communities are at high risk for childhood obesity. The purpose of this manuscript was to review existing childhood and family obesity interventions targeting Appalachian and rural communities to explore recommendations for intervention development. Peer-reviewed studies were selected through a systematic literature search. Seventeen studies met inclusion criteria. Cultural, economical and geographical characteristics of rural and Appalachian communities often make it difficult to reduce childhood obesity. Future research should aim to promote sustainable solutions to complications of childhood obesity in rural and Appalachian communities.
\end{abstract}

Keywords Obesity, Rural, Prevention, Children, Families

\section{Introduction}

One of the overarching goals of Healthy People 2020 is to achieve health equity, eliminate disparities, and improve the health of all groups [1]. Currently in the United States, certain populations, such as those residing in rural and Appalachian areas, have a higher risk for morbidity and mortality, specifically as related to obesity and the subsequent consequences. Beyond the individual consequences of obesity, economic costs of obesity impact the entire nation. As a nation, obesity-related medical costs were estimated at $\$ 147$ billion dollars in 2008 [2]. Considering the costs, both at the individual and societal level, it is important to truly understand the determinants of obesity in high-risk populations in order to make significant changes in these outcomes. There is a growing need for further development, dissemination and evaluation of targeted intervention strategies.

Adult overweight and obesity rates have continued to rise in the U.S., currently peaking at 35.7\% [2]. Twenty years ago, no state had adult obesity rates above $15 \%$. In 2010 , only Colorado had obesity rates lower than $20 \%$ [3], yet there are now 12 states with adult obesity rates higher than $30 \%$. The increase in obesity rates among children may be even more concerning. Over the past three decades, the prevalence of childhood and adolescent obesity has nearly tripled [4]. Among children and adolescents ages 2-19 years, close to $17 \%$ are obese [4]. Eight states in the U.S. have childhood obesity rates above 20\% [3]. Disparities continue to exist, with Appalachian and rural communities reporting some of the highest obesity rates.

According to the Appalachian Region Commission [5], Appalachia refers to the area following the Appalachian mountains and includes all of West Virginia as well as portions of 12 other states: Alabama, Georgia, Kentucky, Maryland, Mississippi, New York, North Carolina, Ohio, Pennsylvania, South Carolina, Tennessee, and Virginia. A large portion of this region is rural. Rural, by the U.S. Census Bureau [6], is considered anything not urban, or a population smaller than 2,500 people. A study by Patterson and colleagues revealed that rural areas are more likely to report having lower health status and higher rates of obesity [7]. Children are uniquely disproportionate in rates of obesity by location. Rural children differ from urban children, $16.5 \%$ to $14.4 \%$, respectively, in prevalence of obesity [7]. Children in rural areas are about $25 \%$ more likely to be overweight or obese than children living in metropolitan areas [8].

Obesity in childhood increases likelihood for adult obesity, which is associated with numerous health risks [9]. Children who are obese are at increased risks for high blood pressure and high cholesterol, type 2 diabetes, respiratory problems, joint and musculoskeletal issues, liver disease and other intestinal complications [9]. Individuals living in rural and Appalachian communities report increased rates of diabetes [10], along with higher risk for morbidity and mortality.

In addition to the physiological complications of obesity, obese children may experience increased rates of psychosocial disorders [11]. Children who are overweight are at higher risk for depression, social withdrawal and may 
also have lower self-esteem than normal weight children [11]. Children have reported social discrimination and bullying as results of their weight status [12]. In general, obese children reportedly experience a lower quality of life due to the combined physical limitations of not being able to participate in sports and other physical activities, and emotional distress stemming from discrimination, teasing, and low self-esteem [12].

Reasons for the rising rates of obesity in rural and Appalachian children are not concrete, but may be related to several factors including: poverty $[13,14]$, inadequate access to healthcare and preventive health $[14,15]$, limited financial resources [16], low levels of physical activity [8], poor nutrition practices [17], and lack of supportive environments. Understanding the determinants related to obesity in these areas is critical when developing and evaluating targeted interventions.

Poverty is a well-recognized risk factor for obesity and related health complications [15]. Adults earning less than $\$ 15,000$ per year had a $33 \%$ prevalence of obesity contrasted by only $24.6 \%$ among those earning $\$ 50,000$ or more per year [6]. In 2011, according to the U.S. Census Bureau [18], 12 states earned a median income of less than $\$ 45,000$ per year. Of those, 7 states were part of the Appalachian Region of the U.S. [5]. The poverty rate of this region was $18 \%$ more than the national average in 2009 [19]. Children of low-income families also tend to have less access to preventive and emergency care and are affected more frequently by poor nutrition and lack of safe, adequate play space [15]. Rural children were less likely to be covered by health insurance, and nearly $50 \%$ less likely to have been seen by a physician for preventative care [8]. Rural areas may also experience inequalities in local government financial resources. Local governments may be limited to programs that are required and may not have funding available to address childhood obesity issues [16]. For children of these low-income families, obesity prevention education and intervention is particularly important due to the potential lack of medical, or health-related formal communication, in the surrounding community.

Additional factors that may contribute to the obesogenic rural setting include lack of access to healthy foods, or "food deserts", higher cost of fruits vegetables, fewer health education opportunities, few opportunities for physical activity, cultural norms reinforcing unhealthy practices, and decreased access to preventative care like dieticians [8]. As reinforced by the Surgeon General's Report, for a large majority of people, overweight and obesity result from excess energy consumption and/or inadequate physical activity. [20] Unhealthy eating habits and physical inactivity are often established early in life and may contribute to the development of childhood overweight and obesity.

Modification of diet tends to be the cornerstone of strategies aimed at preventing and controlling childhood overweight and obesity. [21] However, the propensity for unhealthy nutrition practices in Appalachian and rural areas cannot be ignored due to the ingrained cultural influences and within family food patterns. Often times, a traditional Appalachian diet will include high fat foods such as gravy, fatty meats, and fried foods. [22] Compared to national averages, Appalachian areas may consume fruits and vegetables six fewer times per week. [22] Sunday dinners are a common practice and tend to be very large meals, including biscuits and gravy, several variations of meat (often prepared by frying, stewing, or roasting), several types of vegetables dishes, pies, and milk. [22] Family plays a critical factor in the majority of food choices and nutrition behaviors of children and needs to be further understood. [23]

\subsection{Purpose}

Considering the unique characteristics of rural and Appalachian communities, there is a need for evidence-based strategies to target the high prevalence of obesity and the associated consequences. Therefore, the purpose of this manuscript was to review existing childhood and family obesity interventions targeting Appalachian and rural communities that were published between 1963 and April 2012. Recommendations to enhance interventions among Appalachian and rural communities are discussed.

\section{Methods}

\subsection{Study Abstraction}

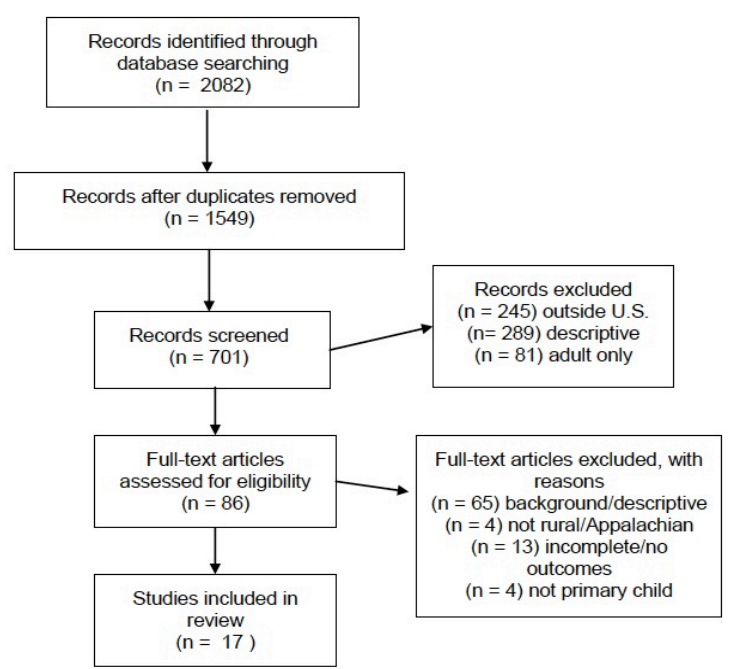

Figure 1. Summary of search

An extensive literature search was conducted independently by two researchers to collect studies for inclusion in this review to increase the likelihood that all pertinent articles were retrieved. Searches were performed using the databases Academic Search Premier, CINAHL, ERIC, Medline, Psychology and Behavioral Sciences Collection, and SPORTDiscus. Various combinations of the following keywords were used: [overweight OR obesity] AND [rural OR Appalachian] AND [program, prevention, 
treatment, intervention, OR study]. Limits of scholarly journals (peer reviewed) were set. Over 2,000 articles were originally identified using these search criteria See the PRISMA flow diagram in Figure 1 for a summary of the systematic search results.

\subsection{Inclusion/Exclusion Criteria}

Inclusion criteria in this review were: (1) publication in the English language, (2) a primary research article evaluating any childhood obesity intervention (prevention or treatment) in rural or Appalachian communities in the U.S., (3) publications in peer reviewed journals between 1963 and April 2012. Exclusion criteria were articles in languages other than English, studies conducted outside of the U.S., case studies, interventions only targeting adults, and those articles which did not present outcomes/findings.

\subsection{Data Extraction}

Data from the studies were extracted independently by two researchers using a standardized form developed by the authors. Any disagreements were examined and the agreed final data recorded. Extracted data included: lead author, publication year, summary of participants, theoretical framework used to guide intervention design and implementation, research design, outcomes, measures used to obtain collected data, description of intervention, intervention frequency and duration, and main findings. These variables are summarized in Tables 1 (children) and 2 (families). 
Table 1. Summary of Child Obesity Prevention/Treatment Interventions

\begin{tabular}{|c|c|c|c|c|c|c|c|}
\hline Author/Year & $\begin{array}{l}\text { Population/ } \\
\text { Sample }\end{array}$ & Design & $\begin{array}{l}\text { Interventio } \\
\text { n Duration/ } \\
\text { Dosage }\end{array}$ & $\begin{array}{l}\text { Intervention } \\
\text { Strategies }\end{array}$ & Theory-based & Outcome Variable(s)/ Measures & Summarized Findings \\
\hline $\begin{array}{c}\text { Canavera et al., } \\
2009\end{array}$ & $\begin{array}{c}\text { 5th grade students } \\
(10-12 \text { years); KY: } \mathrm{N}= \\
122\end{array}$ & Non-experimental & $\begin{array}{l}12 \text { weeks: } 1 \\
\text { session per } \\
\text { week }\end{array}$ & $\begin{array}{c}\text { School-based teacher-delivered } \\
\text { intervention. Focused on PA, FV } \\
\text { consumption, TV usage, and } \\
\text { sweetened beverage consumption. } \\
\text { Student PA sheets and } \\
\text { informational sheets were } \\
\text { provided. } \\
\end{array}$ & $\begin{array}{l}\text { Social } \\
\text { Cognitive } \\
\text { Theory }\end{array}$ & $\begin{array}{l}\text { Baseline and } 12 \text { weeks: } \\
\text { Behaviors: FV consumption, } \\
\text { water consumption, TV } \\
\text { viewing time, and PA. } \\
\text { Expectations, self-efficacy, and } \\
\text { self-control for each } \\
\text { of the four behaviors. } \\
\end{array}$ & $\begin{array}{l}\text { Intervention was successful in } \\
\text { influencing water consumption } \\
\qquad(P<0.05, M=3.99)\end{array}$ \\
\hline $\begin{array}{l}\text { Cottrell et al., } \\
\quad 2005\end{array}$ & $\begin{array}{c}\text { Kindergartners }(4-6 \\
\text { years); WV; } 31 \% \\
\text { overweight or at risk: } \\
\mathrm{N}=50 \text { parent-child } \\
\text { dyads; Control }=26 ; \\
\text { Intervention }=24 \\
\end{array}$ & $\begin{array}{l}\text { RCT, cluster (by } \\
\text { school) }\end{array}$ & 4 weeks & $\begin{array}{l}\text { CARDIAC-Kinder Care: } \\
\text { Pedometers and step logs were } \\
\text { given to participants. Educational } \\
\text { materials provided related to diet, } \\
\text { PA, and BMI. }\end{array}$ & No & $\begin{array}{l}\text { Baseline and } 4 \text { weeks: Child } \\
\text { and parent PA, child diet, and } \\
\text { parental perceptions of } \\
\text { children's PA, diet, and BMI. }\end{array}$ & $\begin{array}{l}\text { Children in treatment group sig. } \\
\text { increased steps taken across } 4 \\
\text { weeks compared to control } \\
(P<0.05) \text {; consumed more } \mathrm{FV} \text { and } \\
\text { fewer sweets on average } \\
\text { compared to control. }\end{array}$ \\
\hline $\begin{array}{l}\text { Drummond et } \\
\text { al., } 2009\end{array}$ & $\begin{array}{l}\text { Childcare centers; rural } \\
\text { AZ: } 30 \text { centers; Appr. } \\
1,800 \text { children }\end{array}$ & Non-experimental & $\begin{array}{l}\text { Overall } \\
\text { 3-years; } \\
\text { varied by } \\
\text { center: } 7 \\
\text { workshops } \\
\text { across } 9 \\
\text { months }\end{array}$ & $\begin{array}{l}\text { Steps to a Healthier Arizona - } \\
\text { Childcare setting: Workshops } \\
\text { given for child-care providers. } \\
\text { Created action plans. Parents } \\
\text { invited to participate in select } \\
\text { workshops. }\end{array}$ & 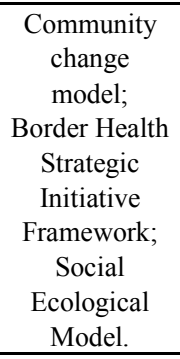 & $\begin{array}{l}\text { Baseline and } 9 \text { months } \\
\text { post-intervention: } \\
\text { Implementing nutrition and PA } \\
\text { best practices in the child care } \\
\text { setting. }\end{array}$ & $\begin{array}{l}\text { Median total number of best } \\
\text { practices sig. increased from } 36 \text { to } \\
44,(P=.0003) \text {; nutrition best } \\
\text { practices increased sig. from } \\
25-30(P=0.0003)\end{array}$ \\
\hline $\begin{array}{c}\text { Gombosi et al., } \\
2007\end{array}$ & $\begin{array}{l}\text { K-8th (5-14 years) } \\
\text { rural PA: School } \\
\text { cohort: } \mathrm{N}=4241+/- \\
200 \text { students each year; }\end{array}$ & $\begin{array}{l}\text { Non-experimental } \\
; \text { longitudinal }\end{array}$ & $\begin{array}{l}\text { Program } \\
\text { was } \\
\text { implemente } \\
\text { d during the } \\
\text { school year } \\
\text { every year } \\
\text { from } \\
\text { 1999-2004. }\end{array}$ & $\begin{array}{l}\text { Fit for Life: Development of a } \\
\text { standard health curriculum for } \\
\text { grades K-8. }\end{array}$ & No & $\begin{array}{l}\text { Follow-up data was collected } \\
\text { yearly: BMI; Implementation } \\
\text { of health education curriculum. }\end{array}$ & $\begin{array}{l}\text { Overall increase in overweight } \\
\text { and obesity of children entering } \\
\text { kindergarten and leaving 8th grade } \\
\text { over the course of the study. }\end{array}$ \\
\hline $\begin{array}{c}\text { Greening et al., } \\
2011\end{array}$ & $\begin{array}{c}\text { 6-10 years; MS; } \\
\text { Control 63\% AA, } \\
\text { Intervention } 58 \% \text { AA: } \\
\mathrm{N}=450 ; \text { Control = } \\
246 ; \text { Intervention }= \\
204\end{array}$ & $\begin{array}{l}\text { RCT, cluster (by } \\
\text { school) }\end{array}$ & 8 months & $\begin{array}{l}\text { TEAM Mississippi Project: } \\
\text { Parental involvement, } \\
\text { environmental changes to school } \\
\text { food. }\end{array}$ & $\begin{array}{l}\text { Social } \\
\text { Learning } \\
\text { Theory }\end{array}$ & $\begin{array}{c}\text { Baseline and } 8 \text { months: } \\
\text { Adiposity measures, Nutrition } \\
\text { knowledge and behaviors, PA }\end{array}$ & $\begin{array}{c}\text { Sig. decline in } \% \text { body fat }(P= \\
0.02) \text {; engaging in sig. more } \mathrm{PA} \\
(P=0.04) \text {; sig. improvement in } \\
\text { dietary fat intake }(P<0.0005) \text {; } \\
\text { sig. improvement } \mathrm{r} \text { performance } \\
\text { on } 2 / 3 \text { fitness tests, including } \\
\text { curl-ups }(P<0.0001) \text {, and shuttle } \\
\text { run }(P<0.0001) \text {. }\end{array}$ \\
\hline Harrell, 2005 & $\begin{array}{l}\text { 5th grade students; } \\
\text { MS: } \mathrm{N}=205\end{array}$ & $\begin{array}{c}\text { Quasi- } \\
\text { experimental }\end{array}$ & $\begin{array}{c}16 \text { weeks; } 4 \\
\text { sessions }\end{array}$ & $\begin{array}{l}\text { Cardiovascular Health in } \\
\text { Children: Classroom sessions on } \\
\text { nutrition and PA; school-wide }\end{array}$ & No & $\begin{array}{c}\text { Baseline and } 16 \text { weeks: Child } \\
\text { and parent FV intake, Health } \\
\text { knowledge }\end{array}$ & $\begin{array}{l}\text { Knowledge sig. increased from } 48 \\
\quad \pm 12 \% \text { to } 60 \pm 14 \% \text { in the } \\
\text { intervention school }(P<0.0001)\end{array}$ \\
\hline
\end{tabular}




\begin{tabular}{|c|c|c|c|c|c|c|c|}
\hline & & & & changes; family component. & & & $\begin{array}{l}\text { reduction in mean energy intake in } \\
\text { both groups }(P<0.0001) \text {; increase } \\
\text { in vegetable consumption in } \\
\text { intervention group }(P<0.05) \text {; } \\
\text { decrease in soft drink } \\
\text { consumption in intervention group } \\
\quad(P<0.05) \text {. }\end{array}$ \\
\hline $\begin{array}{l}\text { Henes et al., } \\
2010\end{array}$ & $\begin{array}{c}2-20 \text { years; } \mathrm{NC} ; \\
\text { Overweight/obese: } \\
\text { N=109 }\end{array}$ & Non-experimental & $\begin{array}{l}\text { Approx } \\
\text { 6-months: } \\
\text { 60-minute } \\
\text { initial } \\
\text { session, } \\
\text { followed by } \\
\text { 45-minute } \\
\text { sessions } \\
\text { scheduled } \\
\text { 2-12 weeks. }\end{array}$ & $\begin{array}{l}\text { KIDPOWER: Patient provideer: } \\
\text { Registered dieticians delivered } \\
\text { medical nutrition therapy to } \\
\text { patients in anywhere from 3-10 } \\
\text { visits. }\end{array}$ & No & $\begin{array}{l}\text { Follow-up period mean months } \\
\text { between visits } 1 \text { and } 3 \text { is } 3.0 \\
\text { months. : Eating behaviors: } \\
\text { consumption of sugar } \\
\text { sweetened beverages, eating at } \\
\text { least five FV each day, limiting } \\
\text { meals eaten away from home } \\
\text { and limiting TV time; Weight } \\
\text { status }\end{array}$ & $\begin{array}{l}\mathrm{FV} \text { intake increased from } 0.61 \text { to } \\
0.92 \text { and } 0.56 \text { to } 0.86 \text { servings/day } \\
(P<0.01) \text {; number times eating out } \\
\text { decreased from } 2.14 \text { to } 1.61 \\
(P<0.05) \text {; decreased TV time, on } \\
\text { weekdays, from } 3.02 \text { to } 2.69 \mathrm{~h} / \text { day } \\
(P<0.05) \text {, and weekend days, } 3.56 \\
\text { to } 2.92 \mathrm{~h} / \text { day }(P<0.001) \text {; Soda and } \\
\text { sweetened beverage intake sig. } \\
\text { decreased }(P<0.01) ; \text { Mean BMI z } \\
\text { score decreased }(P<0.01) \text {. }\end{array}$ \\
\hline $\begin{array}{l}\text { Hovland et al., } \\
2010\end{array}$ & $\begin{array}{c}\text { 3rd grade students; } \\
\text { OH, Appalachia: } \\
\text { Control = 16, } \\
\text { Intervention }=122 \\
\end{array}$ & $\begin{array}{l}\text { Quasi-experiment } \\
\text { al }\end{array}$ & $\begin{array}{l}\text { 9-months: } \\
45 \text { lessons }\end{array}$ & $\begin{array}{l}\text { Teachers delivered } \\
\text { FoodMASTER currliculum to } \\
\text { students in their classrooms. }\end{array}$ & No & $\begin{array}{l}\text { Baseline and end of academic } \\
\text { year: Dietary intake }\end{array}$ & $\begin{array}{l}\text { No sig. differences in intake of } \\
\text { macronutrients, specific nutrients, } \\
\text { or food groups. }\end{array}$ \\
\hline $\begin{array}{l}\text { Muth et al., } \\
\quad 2008\end{array}$ & $\begin{array}{l}\text { 4th grade students; } \\
\text { rural NC: } \mathrm{N}=75 ; \\
\text { Intervention }=38 \\
\quad \text { Control }=37\end{array}$ & $\begin{array}{l}\mathrm{RCT} \text {, cluster (by } \\
\text { classrooms) }\end{array}$ & $\begin{array}{l}12 \text { weeks: } 1 \\
\text { hour/week }\end{array}$ & $\begin{array}{l}\text { Improving Meals and Physical } \\
\text { Activity in Children and Teens } \\
\text { (IMPACT) : Integrated in } \\
\text { classroom curriculum, delivered } \\
\text { by high school students Lessons: } \\
20 \text { mins. of PA and } 40 \text { mins. of } \\
\text { nutrition lesson; Weekly HW that } \\
\text { required parent participation. }\end{array}$ & $\begin{array}{c}\text { Social } \\
\text { Cognitive } \\
\text { Theory }\end{array}$ & $\begin{array}{c}\text { Baseline and } 12 \text { weeks: Dietary } \\
\text { intake }\end{array}$ & $\begin{array}{l}\text { Students in the intervention group } \\
\text { increased daily FV servings (from } \\
2.3 \text { to } 2.7)(P=.05) \text {; increased } \\
\text { calcium-rich foods ( } P=0.07) \text {; grain } \\
\text { intake }(P=0.08) \text {; food group } \\
\text { knowledge increased }(P=0.01) \text {. }\end{array}$ \\
\hline $\begin{array}{l}\text { Schetzina et al., } \\
2009,2011\end{array}$ & $\begin{array}{l}\text { 7-10 years old; rural } \\
\text { Appalachia: } \mathrm{N}=114\end{array}$ & Non-experimental & $\begin{array}{l}18 \text { months: } \\
\text { Health } \\
\text { education } \\
\text { included } 6 \\
\text { interactive } \\
\text { lessons } \\
\text { plans. }\end{array}$ & $\begin{array}{c}\text { Winning with Wellness: CBPR, } \\
\text { school-based approach; nutrition } \\
\text { education, alternate food choices; } \\
\text { use of pedometers; } \\
\text { indoor/outdoor walking trails; } \\
\text { in-class PA breaks; BMI } \\
\text { surveillance; staff wellness; } \\
\text { parental materials sent home }\end{array}$ & $\begin{array}{l}\text { Coordinated } \\
\text { School Health } \\
\text { Model }\end{array}$ & $\begin{array}{l}\text { Baseline and end of school } \\
\text { year: BMI data, PA data, } \\
\text { Cafeteria data, Teacher Child } \\
\text { Obesity Survey }\end{array}$ & $\begin{array}{c}\text { Pedometer data confirmed } \\
\text { students were more active at } \\
\text { school after program } \\
\text { implementation }(P<0.001) \text {. Sig. } \\
\text { fewer unhealthy foods were being } \\
\text { offered and purchased by/served } \\
\text { to students after program } \\
\text { implementation }(P<0.001) \\
\text { Healthy food score in } 2009 \text { was } \\
\text { sig. higher, than } 2005(P<.001) \text {; } \\
\text { students were sig. more active at } \\
\text { school in } 2009(P<.001) \text {, with a } \\
\text { mean of } 4644 \text { steps per day in } \\
2009 \text { and an increase of } 1371 \\
\text { steps per day compared to } 2005 \text {. }\end{array}$ \\
\hline Smith et al., & $3^{\text {rd }}$ and $4^{\text {th }}$ grade & Non-experimental & 8 weeks: 1 & Teen mentoring, education and & Theory of & Baseline and 8 weeks: BMI, & Sig. reduction of BMI percentile \\
\hline
\end{tabular}




\begin{tabular}{|c|c|c|c|c|c|c|c|}
\hline 2011 & $\begin{array}{c}\text { students }(8.51-12.0 \\
\text { years); } 15.4 \% \\
\text { overweight, } 34.6 \% \\
\text { obese: } \mathrm{N}=72 ; \text { Control } \\
=37 ; \text { Intervention }=35\end{array}$ & & hour/week & PA at an after-school program & $\begin{array}{c}\text { Planned } \\
\text { Behavior,Ssel } \\
\text { f-Determinati } \\
\text { on Theory, } \\
\text { Social } \\
\text { Cognitive } \\
\text { Theory }\end{array}$ & $\begin{array}{l}\text { behavioral intention, nutritional } \\
\text { knowledge, attitudes toward } \\
\text { eating healthy, self-efficacy } \\
\text { toward eating healthy and PA }\end{array}$ & $\begin{array}{c}(P=.03) \text {. Teen-mentoring group: } \\
\text { Mean change in BMI from pretest } \\
\text { to posttest was }-0.38 \text { percentile } \\
\text { (SD }=0.93) \text {; Sig. increase in } \\
\text { behavioral intention to eat } \\
\text { healthfully }(P=.02) \text {; Increase in } \\
\text { nutritional knowledge }(P=.05) \text {; } \\
\text { Improved attitude toward eating } \\
\text { healthy }(P=.05)\end{array}$ \\
\hline $\begin{array}{l}\text { Tripp et al., } \\
2011\end{array}$ & $\begin{array}{c}5-18 \text { years; } \mathrm{NC} ; \\
\text { overweight/obese: } \mathrm{N}= \\
50\end{array}$ & Non-experimental & $\begin{array}{l}6 \text { months: } \\
1-6 \text { visits }\end{array}$ & $\begin{array}{l}\text { HEAT (Healthy Eating and } \\
\text { Activity Together): Primary-care: } \\
\text { Use of Motivational interviewing, } \\
\text { family education, health risk } \\
\text { measurements }\end{array}$ & $\begin{array}{l}\text { Motivational } \\
\text { interviewing, } \\
\text { stages of } \\
\text { change (parts } \\
\text { of TTM) }\end{array}$ & $\begin{array}{c}\text { Baseline, each visit and at } 6 \\
\text { months: BMI, motivation, and } \\
\text { compliance }\end{array}$ & $\begin{array}{l}\text { Average motivation went down } \\
\text { between } 2 \text { nd and 3rd visits, but } \\
\text { those who continued process } \\
\text { showed maintained motivation; } \\
\text { Average BMI scores dropped by } \\
\text { 4th and 5th visits (small sample) }\end{array}$ \\
\hline
\end{tabular}

Table 2. Summary of Family-based Obesity Prevention/Treatment Interventions

\begin{tabular}{|c|c|c|c|c|c|c|c|}
\hline Author/Year & $\begin{array}{l}\text { Population/ } \\
\text { Sample }\end{array}$ & Design & $\begin{array}{l}\text { Intervention } \\
\text { Duration/ } \\
\text { Dosage }\end{array}$ & $\begin{array}{l}\text { Intervention } \\
\text { Strategies }\end{array}$ & Theory-based & $\begin{array}{l}\text { Outcome Variable(s)/ } \\
\text { Measures }\end{array}$ & Summarized Findings \\
\hline Follansbee-Junger et al., 2010 & $\begin{array}{c}\text { 8-13 years; rural FL; } \\
\text { overweight/obese; } \mathrm{N}= \\
\text { 67; parent-only=24; } \\
\text { family-based=24; } \\
\text { control=19 }\end{array}$ & $\mathrm{RCT}$ & $\begin{array}{c}16 \text { week: } 12 \\
90 \text {-minute } \\
\text { sessions }\end{array}$ & $\begin{array}{l}\text { Health-care setting: } \\
\text { Incorporated version } \\
\text { of the Stoplight } \\
\text { Program to improve } \\
\text { dietary choices; } \\
\text { increase PA using a } \\
\text { pedometer-based step } \\
\text { program; reduce time } \\
\text { spent in sedentary } \\
\text { activities. }\end{array}$ & No & $\begin{array}{c}\text { Baseline, } \\
\text { post-treatment, and } \\
\text { 6-month follow-up: } \\
\text { Child-reported } \\
\text { disordered eating } \\
\text { attitudes and behaviors; } \\
\text { perceptions of peer } \\
\text { victimization; body } \\
\text { image. Parent-report of } \\
\text { parental control over } \\
\text { child's eating. }\end{array}$ & $\begin{array}{l}\text { At 6-month follow-up } \\
\text { children in both the } \\
\text { family-based and } \\
\text { parent-only conditions } \\
\text { exhibited sig. greater } \\
\text { improvements in weight } \\
\text { status than children in the } \\
\text { control. The mean level of } \\
\text { disordered eating attitudes } \\
\text { endorsed by children in the } \\
\text { intervention and control } \\
\text { conditions at all time points } \\
\text { were well below the clinical } \\
\text { cutoff. }\end{array}$ \\
\hline Haire-Joshu et al., 2008 & $\begin{array}{c}\text { Children: } 1-6 \text { year olds; } \\
\text { Parents: majority } 25-29 \\
\text { years; rural MO; } \mathrm{N}= \\
1658 \text { families; Control } \\
=899 ; \text { Intervention = } \\
759\end{array}$ & $\mathrm{RCT}$ & $\begin{array}{c}\text { Average time } \\
\text { between pretest } \\
\text { and posttest was } \\
7 \text { months (range } \\
6-11 \text { months): } \\
\text { Each family } \\
\text { received } 4 \\
\text { home visits. }\end{array}$ & $\begin{array}{l}\text { High } 5 \text { for Preschool } \\
\text { Kids (H5-KIDS) - } \\
\text { Home-based: Visits } \\
\text { addressed areas such } \\
\text { as knowledge, } \\
\text { parental modeling of } \\
\text { FV intake, } \\
\text { noncoercive feeding } \\
\text { practices, and FV } \\
\text { availability. Parents } \\
\text { received materials and } \\
\text { handouts and children } \\
\end{array}$ & $\begin{array}{l}\text { Social Cognitive } \\
\text { Theory }\end{array}$ & $\begin{array}{l}\text { Weight status, Child } \\
\text { and parent FV intake }\end{array}$ & $\begin{array}{c}\text { H5-KIDS parents sig. } \\
\text { improved intake of fruit } \\
\text { alone }(M=0.14, P=0.04) ; \\
\text { combined FV }(M=0.20, P= \\
\text { 0.05); increase in FV } \\
\text { knowledge and availability } \\
\text { of FV within the home } \\
(P=0.01), \text { and decreased use } \\
\text { of noncoercive } \\
\text { child-feeding practices } \\
\text { compared to those in the } \\
\text { control }(P=0.02) .\end{array}$ \\
\hline
\end{tabular}




\begin{tabular}{|c|c|c|c|c|c|c|c|}
\hline & & & & $\begin{array}{c}\text { received sing-a-long } \\
\text { storybook. }\end{array}$ & & & \\
\hline Hawley et al., 2006 & $\begin{array}{l}\text { 6th graders; Hesston, } \\
\mathrm{KS} ; \mathrm{N}=65 \text { (students); } \\
\mathrm{n}=11 \text { (students and } \\
\text { families) }\end{array}$ & Non-experimental & $\begin{array}{c}6 \text { weeks: } 5 \text {, } \\
40 \text {-minute } \\
\text { sessions }\end{array}$ & $\begin{array}{l}\text { School-based sessions } \\
\text { consisted of games } \\
\text { and tasks. Family Fun } \\
\text { Night offered } \\
\text { educational stations } \\
\text { addressing nutrition } \\
\text { and PA, as well as } \\
\text { goal setting, readiness } \\
\text { for change, and } \\
\text { self-efficacy. }\end{array}$ & $\begin{array}{c}\text { Transtheoretical } \\
\text { Model }\end{array}$ & $\begin{array}{l}\text { Baseline and } 6 \text { weeks: } \\
\text { PA, eating behavior, } \\
\text { BMI, fitness } \\
\text { knowledge, stages of } \\
\text { change }\end{array}$ & $\begin{array}{c}\text { Families sig. increased PA } \\
(M=1.44 \text { METs to } M=7.56 \\
\text { METs, } p<.01) \text {; improved } \\
\text { fitness knowledge, } \\
(P<0.001) \text {; moved forward } \\
\text { appr. one-fourth of a stage } \\
\text { for exercise }(M \text { change } \\
\text { score }=0.27) \text { and one-half of } \\
\text { a stage for nutrition }(M \\
\text { change score }=0.45) .\end{array}$ \\
\hline Horodynski et al., 2004 & $\begin{array}{c}M=30 \text { years; Midwest } \\
\text { U.S.; } N=38 \text { caregivers; } \\
\text { Intervention }=19 ; \\
\quad \text { Control }=19\end{array}$ & Quasi-experimental & $\begin{array}{l}\text { 3-weeks: } 3 \text {, } \\
\text { 90-minute } \\
\text { lessons }\end{array}$ & $\begin{array}{l}\text { NEAT (Nutrition } \\
\text { Education Aimed at } \\
\text { Toddlers) - Early } \\
\text { Head Start Programs: } \\
\text { Lessons were } \\
\text { designed to increase } \\
\text { caregivers' awareness } \\
\text { and knowledge of } \\
\text { healthy eating and } \\
\text { feeding practices in } \\
\text { relation to their } \\
\text { toddlers' } \\
\text { development. }\end{array}$ & No & $\begin{array}{l}\text { Mealtime practices: } \\
\text { knowledge of food and } \\
\text { safety, knowledge of } \\
\text { feeding self-regulation, } \\
\text { strategies to introduce } \\
\text { new foods, serving } \\
\text { healthy snacks, } \\
\text { knowledge of } \\
\text { managing family meals } \\
\text { with toddlers, } \\
\text { toddler-parent } \\
\text { interactions }\end{array}$ & $\begin{array}{l}\text { Six months } \\
\text { post-intervention, no } \\
\text { sig. differences were } \\
\text { found between } \\
\text { groups. }\end{array}$ \\
\hline Horodynski et al., 2005 & $\begin{array}{c}\text { Caregivers }=17-45 \\
\text { years }(M=26 \text { years }) \\
\text { Toddlers }(M=19.3 \\
\text { months); } \mathrm{N}=96 \\
\text { parent-toddler dyads; } \\
\text { intervention }=43 ; \\
\text { control }=53\end{array}$ & Quasi-experimental & 6-months & $\begin{array}{l}\text { Nutrition Education } \\
\text { Aimed at Toddlers } \\
\text { (NEAT) - } \\
\text { Group-based nutrition } \\
\text { lessons and } \\
\text { individually } \\
\text { structured } \\
\text { reinforcement } \\
\text { activities. }\end{array}$ & No & $\begin{array}{l}\text { Baseline, } 4 \text { weeks, and } \\
6 \text { months: Toddlers' } \\
\text { feeding self-regulation, } \\
\text { Parent's knowledge } \\
\text { and self-efficacy in } \\
\text { feeding their toddler. }\end{array}$ & $\begin{array}{l}\text { Caregivers in the } \\
\text { intervention group increased } \\
\text { knowledge compared to } \\
\text { control (14.6 vs. 14.2) and } \\
\text { further improved after the } \\
\text { reinforcement activities } \\
\text { (15.0), while the control } \\
\text { group fell back slightly } \\
\text { (13.9). Caregivers' } \\
\text { self-efficacy score increased } \\
\text { slightly from } 4.0 \text { to } 4.1(P< \\
\text { 0.048) between Time } 1 \text { and } \\
\text { Time 3. This increase } \\
\text { occurred in both the } \\
\text { intervention and control } \\
\text { groups. }\end{array}$ \\
\hline Janicke et al, 2008b & $\begin{array}{c}\text { 8-14 years; } \\
\text { overweight/obese: } \\
\text { Rural; } \mathrm{N}=93 ; \\
\text { Family-based = 33; } \\
\text { Parent-only = 34; }\end{array}$ & $\mathrm{RCT}$ & $\begin{array}{c}4 \text { months: } \\
\text { Weekly group } \\
\text { sessions were } \\
\text { held for the first } \\
8 \text { weeks, then } \\
\end{array}$ & $\begin{array}{l}\text { Dietary habits were } \\
\text { addressed via a } \\
\text { version of the } \\
\text { Stoplight Diet; Goal } \\
\text { setting; Pedometer }\end{array}$ & No & $\begin{array}{c}\text { Baseline, } 4 \text { months, and } \\
10 \text { months: Child and } \\
\text { parent BMI; dietary } \\
\text { intake }\end{array}$ & $\begin{array}{l}\text { PO had greater decrease in } \\
\text { mean BMI z score than } \\
\text { WLC condition }(M=0.127) ; \\
\text { Children in PO intervention } \\
\text { showed a mean decrease of }\end{array}$ \\
\hline
\end{tabular}


Prevention and Treatment Interventions

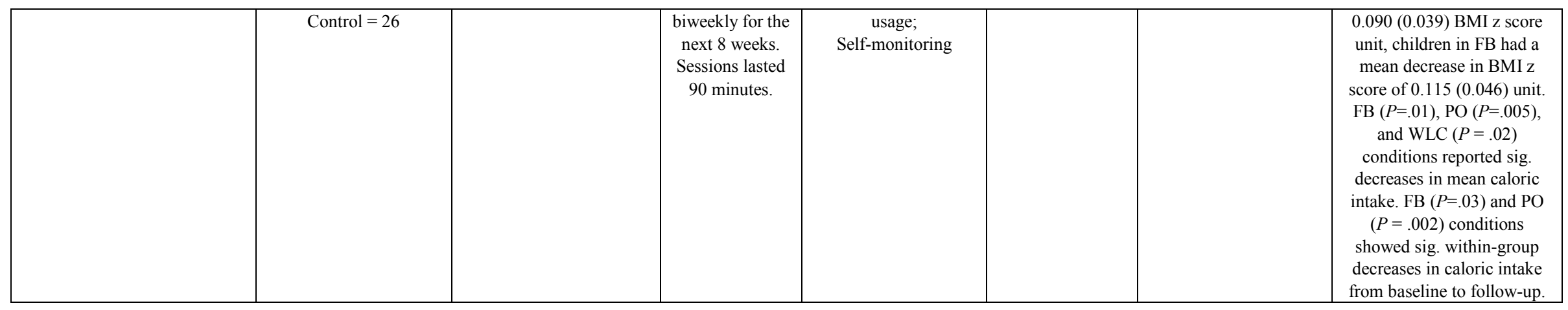




\section{Results}

\subsection{Included Studies}

More than 2,000 articles were identified using the search criteria detailed in the methods. Seventeen interventions were included in this review. See flow diagram in Figure 1 for a summary of the search. The included 17 interventions are summarized in Tables 1 and 2, which give brief descriptions of the target population, interventions and relevant findings, arranged alphabetically by first author's last name.

\subsection{Design and Sample}

Participants' age ranged from 12 months to 17 years old. The largest age group represented in these studies was elementary school children in $\mathrm{K}$ through 6 th grade $(\mathrm{n}=14$, $82 \%$ ) $[14,17]$ [24-35]. Five articles included middle school children (29\%) [17][28][32,33][31], two included high school-age (11.7\%) [28][32], and three included pre-kindergarten children (17.6\%) [13][28][36]. Three programs targeted only parents or caregivers (17.6\%) [13][36,37]. At-risk participants were targeted in six interventions, which included only children, or parents of children, who were overweight or obese (35\%) [25][28][31-33][35].

The number of participants varied by program. One program included less than 50 participants [13], eight included 50-100 participants [25][28,29][31-35], four included 101-400 participants [14][24][27][30], and four interventions studied groups larger than 400 participants [17][26][36,37]. The largest population size was 4,241+/200 students studied by Gombosi et al. for each academic school year from 1999-2004 [17].

Most of the interventions were conducted using a non-experimental study design $(\mathrm{n}=8, \quad 47 \%)$ [17][24][28][30-32][34][37]. Three programs were considered quasi-experimental; a control group was mentioned, however randomization did not occur $[13,14][27]$. Six of the interventions incorporated a randomized control design, including a control group and randomization [25,26][29][33][35,36]. Randomization occurred at the individual/family level for two of the interventions [33][35], the school level or program site for three studies [25,26][36], and by classrooms in one [29].

\subsection{Theoretical Framework}

Theory was used to guide program design and implementation in eight interventions (47\%) [24][26][29-32][34][36]. Constructs of the Social Cognitive Theory, the most commonly used theory, were applied in four interventions [24][29][31][36]. Other theories mentioned were Social Learning Theory [26], Theory of Planned Behavior [31], Self-Determination Theory [31], and The Transtheoretical Model (n=2) [32][34].

\subsection{Intervention Approach}

The most frequently cited setting was school-based $(\mathrm{n}=8$, 47\%) [17][24][26-30][34]. Other settings included homes $(n=2)$ [25][36], early child-care settings $(n=2)$ [13][37], primary health care $(\mathrm{n}=3) \quad[28][32][33]$, and community-based settings $(n=3)$ [13][17][35]. Of the 17 interventions, three addressed only parents or caregivers of young children [13][36][37]. Drummond et al.[37] explored the use of best practices at childcare centers so did not interact directly with children or parents, but rather used an environmental approach to have an indirect impact. Ten interventions included a parental or family component along with strategies aimed at children (59\%) [17][25-27][29,30][32-35]; two interventions used a parent-only experiment group [33][35]; the remaining four interventions included only strategies targeted at children (23.5\%) [14][24][28][31].

Programs ranged in duration from less than 6 weeks $(n=2)[13][25]$ to more than 1 year $(n=3)[14][17][37]$. The largest group of programs $(\mathrm{n}=6,35 \%)$ lasted between 3 and 6 months [13][27,28][32,33][35]. The dosage, or amount of contact, varied widely between programs. The most common dosage of those that indicated this measurement $(n=14,82 \%)$ was less than one contact per week $(n=8,47 \%)$ [27][30][32-34][36,37]. Other programs interacted with participants 1-3 times per week $(n=5)$ [13][24][29][31][35] and more than 3 times per week $(n=1)$ [14].

The most common method of implementation was the use of professionals such as dieticians, pediatricians, nurses, psychologists, etc. to conduct programs and evaluation ( $\mathrm{n}=7$, $41 \%)[13][25,26][30][32][35][37]$. Many programs in the school setting used teaching staff to implement program material $(n=5)$ [14][17][24][26][30]. Teen mentors and parent educators were used in three of the interventions (17.6\%) [29][31][36], and two did not indicate who was responsible for delivering program content $(11.7 \%)[33,34]$.

The programs summarized in Tables 1 and 2 include educational, behavioral, environmental, and social strategies. Eleven interventions used some form of health education strategy (64.7\%) [13,14][17][27-31][34-36]. Educational approaches involved changes to school-based curriculum, either adding a health/nutritional component to physical education classes or incorporating health lessons across disciplines [14][17][27][29,30][33]. Individual or group-based education sessions were also commonly used educational strategies [13][28][32][35,36]. Other educational strategies used were mentoring in an after-school setting [31], and interactive learning activities for parents and children [34]. Interventions using behavioral approaches aimed to change individual behaviors; in many of these cases, dietary practices, physical activity patterns and sedentary/screen time. Fourteen interventions were identified that targeted such individual health behaviors (82\%) [24,25][29-36].

Programs that included environmental approaches $(n=5$, $29 \%$ ) made changes to school-based nutrition programs, care facility best practices, school site health promotion for staff 
and recreation time [26,27][30][33][37]. Some interventions also included social approaches, which generally included a parental component such as family nights or group-based lessons $(\mathrm{n}=4,23.5 \%)[13][26,27][34]$.

\subsection{Intervention Outcomes}

The most common outcome measures were behaviors such as dietary intake (child and/or parent) [14][24-29][34-36], physical activity [24-26][23031][34], and weight status [17][25,26][28][30-32][34-36]. Other outcome measures included screen time [24-28], water consumption [24], nutrition and fitness knowledge [13][26,27][31][34], implementation policies and practices [13][17][37], and expectations, perceptions, and attitudes towards healthy eating and physical activity [24,25][31-33].

Interventions were successful in influencing individual behaviors, improving perceptions and attitudes toward health behaviors, and changing the environment. Overall, 15 interventions were successful to some degree [17][24-37]. Significant improvements in physical activity were identified in four interventions (23.5\%). Cottrell et al. [25] observed significant increases in steps taken for the intervention group compared to the control $(P<.05)$. Greening et al. [26] found significant increases in physical activity $(P=.04)$ as well as significant improvement in performance on two out of three fitness tests, including curl-ups $(P<0.0001)$, and shuttle run $(P<0.0001)$. Schetzina et al. [30] observed that students were more physically active after program implementation $(P<.001)$, and Hawley et al [34] found that families significantly increased physical activity ( $M=1.44$ METs to $M=7.56$ METs, $P<.01)$. Improvement in nutritional behaviors was also noted. For example, Canavera et al. [24] reported increased water consumption among participants $(P<.05)$. Five of the interventions (29\%) found a significant increase in fruit and vegetable consumption post-intervention [25][27-29][36]. Significant improvement in nutrition- and health-related knowledge was reported by four of the interventions (23.5\%) [27][29][31][34]. Weight status or body composition improvements were identified in five programs (29\%), three of these indicating significant changes in body fat [26], BMI [31], or weight status [33].

With regards to environmental changes, Drummond et al. [37] found success in best practices in a childcare setting $(P$ $=.003$ ). In this study, childcare providers implemented changes to organizational practices, policy, and environment to influence positive nutrition and physical activity in young children.

Beyond baseline and post-intervention measures, only four of the studies collected follow-up measures $(23 \%)$, with two completing a 6-month follow-up [13][33], one a 9-month follow-up [37], and one a 10-month follow-up [35].

\section{Discussion}

Childhood obesity is a nationwide concern that disproportionately affects rural and Appalachian areas [7]. The purpose of this manuscript was to review and summarize existing childhood and family obesity prevention and treatment interventions targeting Appalachian and rural communities published between 1963 and April 2012 in order to enhance understanding of the benefits and limitations of strategies used in this population. Findings from these 17 interventions indicate a growing need for programs and strategies to meet the needs of rural and Appalachian children and families. Ages of participants ranged from toddlers to older adolescents, primarily reaching $\mathrm{K}-6$ th grade children. It is important to target children in elementary school because of the crucial eating and physical activity patterns established during these formative years. It is known that patterns developed in youth track into adulthood [34], including relevant health behaviors linked to obesity. In a longitudinal study conducted by Herman et al., $83 \%$ of overweight youth remained overweight as adults [39].

Elementary schools were the setting of $47 \%$ of the resulting interventions. School-based obesity prevention programs have received considerable attention. This is likely due to the existing resources already available in schools and the convenience of having a captive audience for 180 days of the year. Children spend a majority of their hours in school, and all children regardless of their demographics are reached [40]. Schools provide researchers and program planners the opportunity to reach a large population efficiently and effectively due to the capacity of potential staff, environmental means for promoting physical activity and healthy nutrition, and administrative support. Successful school-based programs do not necessarily require the use of additional classroom time, but rather can be integrated into standardized learning and emphasize use of existing personnel and material resources $[29,30]$. Caution should be used when implementing programs into school-based curriculum to ensure that teachers and staff are adequately trained to deliver program materials and that intervention components are being carried out as designed [30].

With the continued promise of school-based interventions, there needs to be a targeted effort to use existing evidence-based guidelines as the foundation for developing, implementing and evaluating obesity prevention interventions. The CDC's Guidelines for Schools to Promote Healthy Eating and Physical Activity reinforce the importance of partnerships among schools, families, and community members. [41] In rural areas specifically, targeting children while at school may help to avoid unique environmental barriers, including transportation and accessibility to nutritious foods, along with health education resources. Due to recent reform regarding school-based nutrition, children, regardless of geographical location, will now have better access to more nutrient-dense, lower-fat foods [42. The USDA has proposed the following reform to school-based nutrition: 
"increase the availability of fruits, vegetables, whole grains, and fat-free and low-fat fluid milk in the school menu; reduce the levels of sodium, saturated fat and trans fat in school meals; and meet the nutrition needs of school children within their calorie requirements".

This beneficial change should prompt schools to discuss healthy nutrition, behavior change goals, and other health promoting topics.

Schools can also promote a sense of community and support for children and their families since they are often used as a community gathering place in rural and Appalachian regions [27]. It is imperative to include the family as a focal point for evoking change in cultural norms, adoption of healthier lifestyles, and overall improved wellness. [21-23] Parents are identified as a child's most important role models [4]. Parental involvement was frequently cited as a critical component in making changes in children's physical activity and eating habits. In the majority of the interventions reviewed, parents were involved at some level [13][17][25-27][30][32-37], though the level of involvement varied across intervention strategies. Parents are in a unique position to directly influence a child's food preferences from a very young age by the foods they offer to the child [36]. Involvement of parents and families in a childhood obesity treatment or prevention program is also important in influencing a child's physical activity. Interventions that included parents showed success in decreasing children's television viewing time [28], increasing child's physical activity [30], increasing child's fruit and vegetable consumption [36], and improving children's weight status [35].

Successful strategies involving parents included the use of parent-only intervention groups that allowed for parent-directed health education and skill building, opportunities for modeling and practicing different feeding behaviors, and inclusion of parents in goal setting and take-home activities. Evidence in one study suggested that parent-only intervention groups might, in fact, be more successful than family-based interventions [35]. One possible explanation is that children who attend weight management programs may feel stigmatized and lose motivation or willingness to comply.

An effective intervention strategy to be incorporated into future interventions may be to relate healthy eating to the overall benefit of the children in the family. [22] As children are highly valued in rural and Appalachian families, this could be the impetus needed to more positively alter some of the cultural ideations related to nutrition and physical activity of the past. Changes in family meals and behaviors may ultimately impact the entire family and surrounding community. [22] Another potential benefit of parent-only interventions is the use of fewer personnel and material resources for program delivery, which is particularly important in resource-poor regions. Future research should explore these challenges in an effort to fully impact obesity rates in rural communities.

It is well documented that use of theory in program design increases the effectiveness and validity of an intervention [43]. Theory was used in $47 \%$ of the included interventions. Of the interventions that used theory, success was shown in areas such as increased water consumption [24], decreased body fat [26], decreased BMI [31,32], increased physical activity [26][30], improved dietary fat intake [26], improved performance on fitness tests [26], increased daily fruit and vegetable consumption [29], increased calcium consumption [29], increased nutritional knowledge [31], and improved attitudes toward nutrition and health eating [31].

Social Cognitive Theory (SCT) was the most commonly used theory in the reviewed interventions. SCT has been used in the development of childhood obesity interventions because it includes constructs that support self-efficacy and self-management of health behaviors [24]. Many health habits are formed in childhood, thus it is more effective to instill healthy behaviors at an early age than to change them throughout the lifespan. Muth et al. [29] drew upon elements of the SCT, noting the reciprocal influence of the school environment on childhood eating and physical activity patterns. In addition, Muth et al. hypothesized that peer modeling may influence children's behaviors. Self-efficacy (for healthy eating and physical activity) was also a commonly measured construct drawn from the SCT [31], as well as behavioral capacity, or knowledge of health-related behaviors [36]. Theory guides program design and implementation, as well as behavior change strategies. Use of theory is a supported method for implementing evidence-based programs, and contributes to the validity and reliability of intervention strategies. Future research is needed to determine appropriate theoretical frameworks to be used within rural and Appalachian communities.

Several successful strategies were employed in the reviewed rural and Appalachian interventions. Teen mentoring was successfully implemented in two interventions [29][31]. Use of teens as mentors is an inexpensive and effective mechanism for working with children and adolescents, especially in areas that may lack formal health education programming. Programs that used teen or peer mentors used strategies such as role playing, parental involvement in the form of take-home assignments, educational opportunities, and opportunities to practice physical activities and build self-efficacy for these activities. Shifting cultural norms in rural communities may be helpful in the treatment and prevention of obesity. Peers often look up to others in their community, thereby creating an integral opportunity to promote healthy lifestyle behaviors.

Other successful interventions used strategies that incorporated provider-patient collaboration [32]. Blizzard Tripp et al.[32] integrated motivational interviewing techniques to evoke behavior change with input from parents and patient. Use of parent and patient collaboration to develop a tailored program was cited as an effective tool in several interventions [13][28-30][32,33][35,36].

Providing opportunities for skill practice was also an important technique for success. Future interventions should build on this strategy and also include tools and training for 
healthcare providers (when applicable) so as to increase their self-efficacy for counseling parents and patients regarding overweight and obesity.

Environmental approaches, such as those employed in schools to change health curriculum, were successful in several interventions through changes to policy and practice within an institution. Changes to the environment in an institution such as a school or childcare facility are far-reaching and are supported by multiple levels of management, thereby increasing the likelihood of enforcement. Strategies such as changes in staff wellness programs, changes in vending options and cafeteria offerings, and changes to facility best practices, were successfully implemented in five interventions $[26,27][30][33][37]$. Recommendations continue to incorporate strategies which affect environmental change, including providing access to healthy foods throughout the school day and establishing a school culture which encourages healthy eating among students and staff. [41] Although more and more interventions are including environmental changes, there is still a need to fully understand feasible and effective strategies. Use of environmental approaches should be further explored in experimental, longitudinal studies to determine effectiveness and sustainability of these programs throughout rural and Appalachian communities.

Intervention success was identified at varying levels of intervention approaches. Tailored, individualized programs were successful in changing attitudes and behaviors, as were environmental, or school-wide intervention approaches. Further research should be conducted to determine the most effective approaches for at risk populations, including those living in rural and Appalachian areas.

\subsection{Limitations}

It is important to note that this is a narrative review and not a quantitative meta-analysis. Interventions in this review were limited to those written in the English language, targeting only rural and Appalachian children in the United States. In addition, rural and Appalachian populations may not have been specified in all manuscripts reviewed, thus this review included only those that were explicitly defined by the intervention authors.

\subsection{Suggestions for Further Research/Future Interventions}

Future childhood obesity prevention and treatment interventions in rural or Appalachian areas should include a parental and/or familial component. This will lead to more sustainable changes in a child's behavior, particularly as it relates to nutrition practices, and thereby reduce the risk of chronic disease. Interventions should also include opportunities for skill-building and practical application in order to build child and parent self-efficacy for behavior change.
Though important changes to school-based nutrition have been put in effect, [42] training and evaluation of school food service staff will be required for effective progress. Improvements in cultural dietary practices may require slow, gradual change and the implementation of techniques such as: gradually reducing consumption of certain foods versus eliminating abruptly, adding high-fiber, nutrient dense foods alongside unhealthier choices or in place of higher fat choices, and adjusting preparation methods rather than overhauling food content. [22] Again, these changes can be incorporated into school-based interventions, family-based interventions, or a combination of the two. Additionally, strategies to identify, recognize, and integrate these cultural norms should be a priority to ultimately determine impact of nutrition practices on obesity prevention interventions in rural and Appalachian populations.

Educating healthcare practitioners serving rural and Appalachian areas is an area needing further investigation. Equipping these providers with necessary skills to address overweight and obese youth patients and their parents increases the likelihood of improved behavior changes to promote obesity prevention. These providers are often community members and may be considered a trusted source within the broader scope of the community.

Program dosage and duration varied widely in these interventions and should be further explored to determine if standards can be established. Long-term follow-up is required in order to determine whether or not programs are sustainable and whether or not there have been significant changes made in the reduction of morbidity and mortality from obesity-related illness. Similarly, most of the interventions did not use an experimental design, limiting the generalizability to other rural and Appalachian areas. Future studies should work to conduct randomized, controlled trials in an effort to distinguish whether changes are due to intervention strategies or extraneous factors.

\section{Conclusion}

It is evident that children growing up in rural and Appalachian regions are faced with environmental and social barriers that challenge their health and limit their access to health education. The geographical layout of rural communities, cultural cooking practices, and lack of access to quality healthcare contribute to an increased risk for overweight and obesity among children in these areas. As health professionals, it is imperative to design and promote evidence-based programs targeted towards improving the eating environment in Appalachian homes and schools, involving parents in children's dietary and physical activity choices, and increasing physician involvement in addressing overweight and obesity-related health concerns. Protecting the health of at-risk children will help sustain improvements to our nation's healthcare burden, decrease health disparities, and improve quality of life for individuals across all ages and demographics. 


\section{REFERENCES}

[1] United States Department of Health and Human Services. About Healthy People 2020, Healthy People 2020 Web site, 2012. Online available: http://www.healthypeople.gov/2020/ about/default.aspx.

[2] Centers for Disease Control and Prevention. Overweight and obesity: adult obesity facts. Centers for Disease Control and Prevention Web site. Online available: http://www.cdc.gov/o besity/data/adult.html.

[3] Robert Wood Johnson Foundation. Trust for America's Health. F as in fat: how obesity threatens America's future 2011, Robert Wood Johnson Foundation, 2011. Online available: http://www.healthyamericans.org/report/88/.

[4] Centers for Disease Control and Prevention. Overweight and obesity: childhood obesity facts. Centers for Disease Control and Prevention Web site. Online available: http://www.cdc.gov/obesity/data/childhood.html.

[5] Appalachian Regional Commission. The Appalachian region. Online available: http://www.arc.gov/appalachian_region/Th eAppalachianRegion.asp.

[6] U.S. Department of Health and Human Services, Health Resources and Services Administration. Defining the rural population. Online available: http://www.hrsa.gov/ruralhealt $\mathrm{h} /$ policy/definition_of_rural.html.http://www.healthyamerica ns.org/report/88/

[7] P. Patterson, C. Moore, J. Probst, J. Shinogle. Obesity and physical inactivity in rural America, Journal of Rural Health, Vol. 20, 151-159, 2004.

[8] M. Lutfiyya, M. Lipsky, J. Wisdom-Behounek, M. Inpanbutr-Martinkus. Is rural residency a risk factor for overweight and obesity for U.S. children?, Obesity, Vol. 15, No. 9, 2348-2356, 2007

[9] Centers for Disease Control and Prevention. Overweight and obesity: basics about childhood obesity. Centers for Disease Control and Prevention Website. Online available: http://www.cdc.gov/obesity/childhood/basics.html.

[10] Centers for Disease Control and Prevention. Obesity, diabetes estimates by county, 2007. Online available: http://www.cdc.gov/Features/dsObesityDiabetes/index.html.

[11] S. Erermis, N. Cetin, M. Tamar, N. Bukusoglu, F. Akdeniz, D. Goksen. Is obesity a risk factor for psychopathology among adolescents?, Pediatric International, Vol. 46, 296-301, 2004. doi: 10.1111/j.1442-200x.2004.01882.x.

[12] L. Marcus, A. Baron. Childhood obesity: the effects on physical and mental health. New York, NY, NYU Child Study Center, n.d. Online available: http://www.aboutourkid s.org/articles /childhood_obesity_effects_physical_mental_h ealth.

[13] M. Omar Horodynski, S. Hoerr, G. Coleman. Nutrition education aimed at toddlers: a pilot program for rural, low-income families, Family and Community Health, Vol. 27, No. 2, 103-113, 2004.

[14] J. Hovland, M. McCleod, M. Duffrin, G. Johanson, D.
Berryman. School-based screening of the dietary intakes of third graders in rural Appalachian Ohio, Journal of School Health, Vol. 80, No. 11, 536-543, 2010.

[15] D. Wood. Effect of child and family poverty on child health in the United States, Pediatrics, Vol. 112, 707-711, 2003. Online available:http://www.pediatricsdigest.mobi/content/1 12/Supplement_3/707.short.

[16] National Association of Counties (NACo). Rural Obesity: Strategies to Support Rural Counties in Building Capacity, Washington, DC: NACo, 2008. Online available: http://www.cdc.gov/CommunitiesPuttingPreventiontoWork/r esources/obesity.htm.

[17] R. Gombosi, R. Olasin, J. Bittle. Tioga County Fit for Life: a primary obesity prevention intervention, Clinical Pediatrics, Vol. 46, No. 7, 592-600, 2007.

[18] United States Census Bureau. Income: state median income. U.S. Census Bureau Web site. Online available: http://www.census.gov/hhes/www/income/data/statemedian/.

[19] Appalachian Regional Commission. Economic reports: Appalachian region income reports-2011. Online available: http://www.arc.gov/research/EconomicReports.asp.

[20] The Office of the Surgeon General. The surgeon general's call to action to prevent and decrease overweight and obesity .Office of the Surgeon General (Ed.). Rockville, MD, 2001.

[21] M. Sharma. Dietary education in school-based childhood obesity prevention programs. Advanced Nutrition, 2, 207s-216s, 2011.

[22] W. Flasher. Cultural diversity: Eating in America, Appalachian. Ohio State University Family and Consumer Science. Online available http://ohioline.osu.edu/hyg-fact $/ 5$ 000/pdf/5252.pdf

[23] G. Tabacchi, S. Giammanco, M. La Guardia, M. Giammanco. A review of the literature and a new classification of the early determinants of childhood obesity: From pregnancy to the first years of life. Nutrition Research, 27, 587-604, 2007.

[24] M. Canavera, M. Sharma, J. Murnan. Development and pilot testing a social cognitive theory-based intervention to prevent childhood obesity among elementary students in rural Kentucky. International Quarterly of Community Health Education. Vol. 29, No. 1, 57-70, 2009.

[25] L. Cottrell, E. Spangler-Murphy, V. Minor, A. Downes, P. Nicholson, W. Neal. A kindergarten cardiovascular risk surveillance study: CARDIAC-Kinder, American Journal of Health Behavior, Vol. 29, No. 6, 595-606, 2005.

[26] L. Greening, K. Harrell, A. Low, C. Fielder. Efficacy of a school-based childhood obesity intervention program in a rural southern community: TEAM Mississippi Project, Obesity, Vol. 19, No. 6, 1213-1219, 2011.

[27] T. Harrell, B. Davy, J. Stewart, D. King. Effectiveness of a school-based intervention to increase health knowledge of cardiovascular disease risk factors among rural Mississippi middle school children. Southern Medical Journal, Vol. 98, No. 12, 1173-1180, 2005.

[28] S. Henes, D. Collier, S. Morrissey, D. Cummings, K. Kolasa. Medical nutrition therapy for overweight youth in their medical home: the KIDPOWER experience, Patient 
Education and Counseling, Vol. 81, 43-46, 2010.

[29] N. Muth, A. Chatterjee, D. Williams , A. Cross, K. Flower. Making an IMPACT: effect of a school-based pilot intervention. North Carolina Medical Journal, Vol. 69, No. 6, 433-440, 2008.

[30] K. Schetzina, W. Dalton, D Pfortmiller, H. Robinson, E. Lowe, H. Stern. The Winning with Wellness project: rural Appalachian elementary student physical activity and eating behavior and program implementation 4 years later, Family and Community Health, Vol., 34, No. 2, 154-162, 2011.

[31] L. Smith. Piloting the use of teen mentors to promote a healthy diet and physical activity among children in Appalachia, Journal for Specialists in Pediatric Nursing, Vol., 16, 16-26, 2011.

[32] S. Blizzard Tripp, J. Templeton Perry, S. Romney, J. Blood-Siegfried. Providers as weight coaches: using practice guides and motivational interview to treat obesity in the pediatric office, Journal of Pediatric Nursing, Vol. 26, 474-479, 2011.

[33] K. Follansbee-Junger, D. Janicke, B. Sallinen. The influence of a behavioral weight management program on disordered eating attitudes and behaviors in children with overweight, Journal of the American Dietetic Association, Vol. 110, No. 11, 1653-1659, 2010.

[34] S. Hawley, H. Beckman, T. Bishop. Development of an obesity prevention and management program for children and adolescents in a rural setting, Journal of Community Health Nursing, Vol. 23, No. 2, 69-80, 2006.

[35] D. Janicke. B. Sallinen, M. Perri. Comparison of parent-only vs family-based interventions for overweight children in underserved rural settings, Archives of Pediatric and
Adolescent Medicine, Vol. 162, No. 12, 1119-1125, 2008.

[36] D. Haire-Joshu, M. Eilliott, N. Caito, et al. High 5 for Kids: the impact of a home visiting program on fruit and vegetable intake of parents and their preschool children, Journal of Preventive Medicine, Vol. 47, 77-82, 2008.

[37] R. Drummond, L. Staten, A. Reyes Sanford, et al. A pebble in the pond: the ripple effect of an obesity prevention intervention targeting the child care environment, Health Promotion Practice, Vol. 10, No. 2, 156S-167S, 2009.

[38] J. Savage, J. Fisher, L. Birch. Parental influence on eating behavior: conception to adolescence, Journal of Law, Medicine, \& Ethics, Vol. 35, 22-34, 2007.

[39] K. Herman, C. Craig, L. Gauvin, P. Katzmarzyk. Tracking of obesity and physical activity from childhood to adulthood: the physical activity longitudinal study, International Journal of Pediatric Obesity, Vol. 4, 281-288, 2009.

[40] R. Goutham. Childhood obesity: highlights of AMA expert committee recommendations, American Family Physician, Vol. 78, 56-64, 2008.

[41] Centers for Disease Control and Prevention. School health guidelines to promote healthy eating and physical activity. Morbidity and Mortality Weekly Report, 60, 1-76, 2012.

[42] United States Department of Agriculture, Food and Nutrition Services. Nutrition standards for school meals. United States Department of Agriculture website. Online available: http://www.fns.usda.gov/cnd/governance/legislation/nutritio nstandards.htm

[43] K. Glanz, B. Rimer, K. Viswanath. Health Behavior and Health Education: Theory, Research, and Practice, 4th ed. San Francisco, CA: Jossey-Bass, Inc., 2008. 